\title{
VCAM (CD-106) and ICAM (CD-54) Adhesion Molecules Distinguish Keratoacanthomas from Cutaneous Squamous Cell Carcinomas
}

\author{
Nicole D. Melendez, B.S., Bruce R. Smoller, M.D., Michael Morgan, M.D. \\ Department of Pathology (N.D.M., M.M.), University of South Florida, Tampa, Florida, and James A. \\ Haley Veterans Administration Hospital; and the Departments of Pathology and Dermatology (B.R.S.), the \\ University of Arkansas for Medical Sciences, Little Rock, Arkansas
}

Keratoacanthomas are rapidly growing benign epithelial derived neoplasms that may evolve into squamous cell carcinomas, or represent a variant of squamous cell carcinoma. ICAM (CD-54) is a ligand for the cell adhesion receptor LFA-1, shown to be important in immune stimulation that is upgraded in inflammatory cutaneous disorders. VCAM (CD106) is an adhesion molecule normally found in stimulated endothelium, that plays a critical role in the migration of leukocytes. We examined the immunohistochemical expression of ICAM (CD-54) and VCAM (CD-106) in a series of 50 evolving, fully developed, resolving keratoacanthoma and welldifferentiated and poorly differentiated squamous cell carcinoma to evaluate the possible temporal and pathogenic relation of these immune recognition markers and epithelial derived tumors. ICAM (CD-54) showed an increase in expression in the fully developed keratoacanthoma and was absent in the evolving and resolved keratoacanthoma. In the squamous cell carcinomas, expression was focally observed in the well-differentiated squamous cell carcinomas with a dramatic increase seen in the poorly differentiated squamous cell carcinomas. Similarly, VCAM (CD-106) was expressed in the fully developed keratoacanthoma and was absent in the evolving and resolved keratoacanthoma. Moderate expression for VCAM (CD-106) was seen in the welldifferentiated squamous cell carcinoma, and intense expression was seen in the fully developed keratoacanthoma and poorly differentiated squamous cell carcinoma. As a group, keratoacanthoma and squamous cell carcinoma are immunophenotypically distinct. There is a temporal related

\footnotetext{
Copyright () 2003 by The United States and Canadian Academy of Pathology, Inc.

VOL. 16, NO. 1, P. 8, 2003 Printed in the U.S.A.

Date of acceptance: October 1, 2002.

Address reprint requests to: Michael B. Morgan, M.D., 16124 Chastain

Road, Odessa, FL 33556; e-mail: mbkmmorgan@aol.com.

DOI: 10.1097/01.MP.0000043520.74056.CD
}

increase in expression of VCAM (CD-106) in conjunction with the evolution of keratoacanthoma. Increased expression of both markers is seen with squamous cell carcinoma dedifferentiation. Application of these markers might be an important adjunct in predicting the biologic behavior and pathogenesis of these lesions.

KEY WORDS: Keratoacanthoma, squamous cell carcinoma, VCAM, ICAM

Mod Pathol 2003;16(1):8-13

Keratoacanthoma are rapidly growing, crater-like neoplasms of the skin that typically affect hairy, sunexposed areas, especially the face, and dorsum of the arms and hands (1). These are more common in fair skinned elderly males. They are cytologically identical to cutaneous squamous-cell carcinomas and it has been debated whether keratoacanthoma is a variant of squamous cell carcinomas or progress into squamous cell carcinoma (2). While the majority of keratoacanthoma are benign and regress without treatment, there have been cases of metastasizing keratoacanthoma further adding to the confusion as to the nature of these neoplasms (3). Because of this it is important to understand the pathogenesis and biologic behavior of keratoacanthoma.

In most instances, keratoacanthomas may be distinguished from squamous cell carcinoma. This distinction can be determined by growth characteristics, subtle histologic differences, and most recently, on differences in the expression of immunologically mediated adhesion molecule expression (4-6). Keratoacanthoma is characterized by rapid growth and involution in distinction to the gradual and inexorable growth of squamous cell carcinoma. Keratoacanthoma forms a symmetrical cup-shaped architecture confined to the dermis; whereas squamous cell carcinoma is typically asymmetrical with a variable architectural silhouette that may be seen extending as infiltrating single cells or tumoral 
islands into deeper levels of the dermis and subcutaneous fat (4). Additional features characteristic of keratoacanthoma seen in the fully developed or resolving stages include the transepidermal elimination of elastic fibers, neutrophilic microabscesses, and eosinophils in the surrounding peritumoral stroma. The latter findings have suggested to some the possibility of a pathogenically distinct host or immunologically mediated response to these tumors (5). This supposition has been underscored by studies that have shown differences in the expression of the surface adhesion molecules syndecan-1 and ICAM (CD-54) and involucrin, a cytoplasmic protein (5-8).

Cell to cell adhesion is regulated by adhesion molecules (9). The adhesion receptors belong to four molecular families, including the selectins, integrins, mucinlike glycoproteins, and immunoglobin family molecules, including ICAM and VCAM. The latter are upregulated by cytokines including interleukin 1 and TNF- $\alpha$ associated with immunologic activation (10-12). ICAM (CD54 ) is expressed on endothelial cells and monocytes and has been shown to be increased in keratoacanthoma and poorly differentiated squamous cell carcinoma (5). VCAM (CD-106) is released from activated endothelium and binds and regulates immune effector cells including neutrophils, lymphocytes, and eosinophils through interaction with integrin receptors (13). The expression pattern and or intensity of VCAM (CD-106) have not been studied in keratoacanthoma or squamous cell carcinoma, to our knowledge.

Given the relative lack of inquiry involving adhesion molecule expression and their ascribed role in the normal immune response, we investigated a series of keratoacanthoma at various stages of development and squamous cell carcinoma of varying histologic grade with specific aims to ascertain 1.) whether keratoacanthoma and squamous cell carcinoma are immunophenotypically distinct; 2.) whether keratoacanthoma shows a difference in the expression of adhesion molecules at the various stages of development; 3.) whether there are differences in the level of adhesion molecule expression between well differentiated and poorly differentiated squamous cell carcinoma; and finally 4.) whether adhesion molecule expression has relevance in the pathogenesis of these lesions.

\section{MATERIALS AND METHODS}

A total of 50 cases of keratoacanthoma and cutaneous squamous cell carcinomas consisting of 10 evolving, 10 fully developed, 10 regressing, 10 well differentiated and 10 poorly differentiated squamous cell carcinoma were obtained from the surgical pathology files at the James A. Haley Veterans Administration Hospital, Tampa, Florida. Evolving keratoacanthoma were defined by the histologic presence of a symmetrical cup shaped proliferation of clear to glassy appearing epithelium associated with a distorted follicular infundibulum. The lesional cells possessed mild nuclear atypia with rare dyskeratotic cells. Fully developed keratoacanthoma consisted of larger more irregular infiltrating squamous nests and islands with increased dyskeratosis and scattered typical and atypical mitotic figures (Fig. 1). The resolving keratoacanthoma consisted of superficial dermal lesions with, scalloped or jagged epithelial remnants punctuated by perforating strands of elastin, dermal fibrosis and squamous syringometaplasia (Fig. 2). Well differentiated squamous cell carcinoma were defined by the presence of asymmetric nests and or single cell dermal invasion by epithelial cells showing cytologic atypia consisting of nuclear hyperchromasia, typical and atypical mitotic figures, tumor necrosis, dyskeratosis and cytoplasmic eosinophilia throughout all levels of neoplasm. Poorly differentiated squamous cell carcinoma consisted of more irregular and infiltrating cords of poorly keratinized epithelial cells with nuclear anaplasia, multinucleation, numerous typical and atypical mitotic figures and/or cytoplasmic spindling.

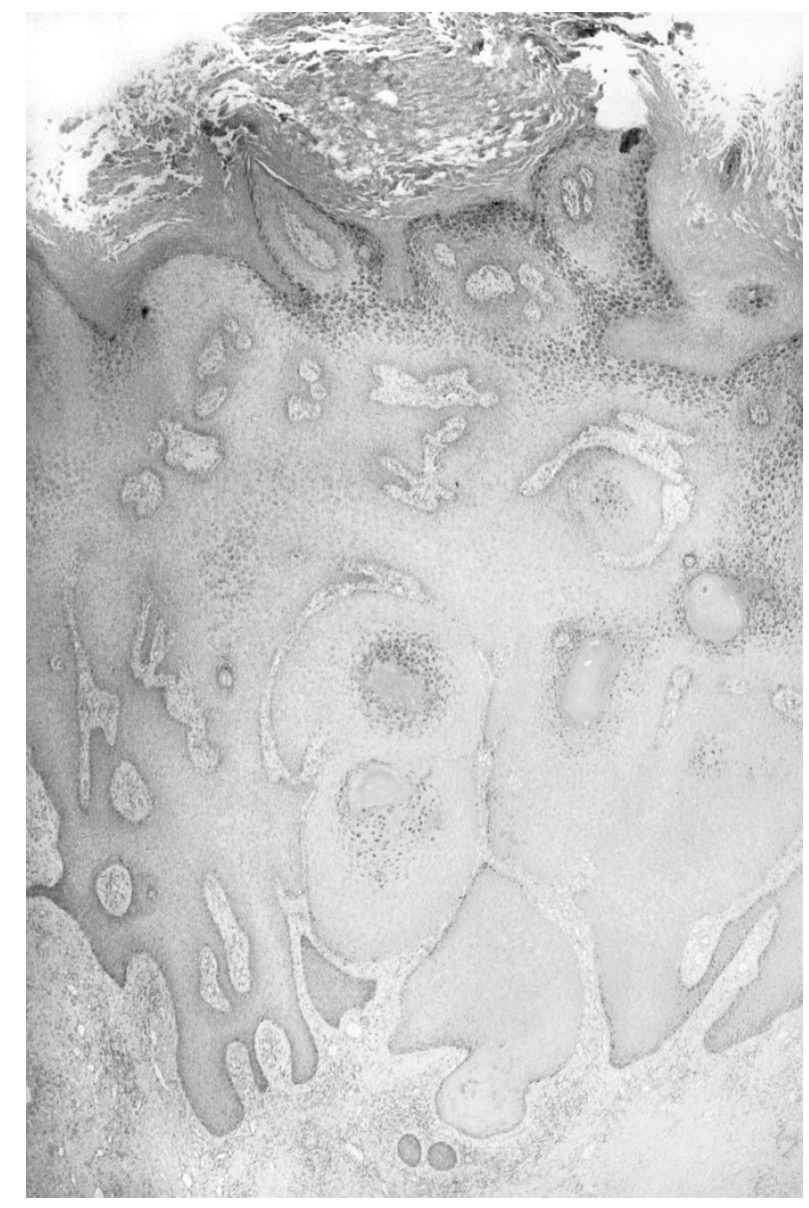

FIGURE 1. Fully developed keratocanthoma consisting of large irregular infiltrating squamous nests and islands $(40 \times)$. 
None of the patients in this study were immunocompromised or possessed the multiple keratoacanthoma or familial syndromes of Ferguson-Smith, Gryzbowski, Zak-Witten or Muir-Torre Syndrome. Rare keratoacanthoma variants including the giant form, subungual, or keratoacanthoma centrifugatum marginatum were not studied. Chart review in all of the cases of keratoacanthoma and squamous cell carcinoma showed no record of metastases or of death due to disseminated disease.

All sections were fixed in formalin and embedded in paraffin. The immunohistochemical stains were ICAM (CD-54; Novocastra Laboratories, United Kingdom, 1:20 dilution) and VCAM (CD-106; Novocastra Laboratories, 1:50 dilution). All of the staining was done according to the manufacturer's instructions using the avidin-biotin complex (ABC) method with appropriate positive and negative controls. The results were interpreted by light microscopy and scored based on the intensity, pattern, and the location of tumor cell(s) stained. Intensity of stain was evaluated independently by two board-certified dermatopathologists (MM, BS) with scores averaged according to whether it was absent $(0)$, weakly $(1+)$, moderately $(2+)$, or strongly positive $(3+)$. The range of immunohistochemical intensity observed for each marker and tumor was calculated. Qualitative assessments of the percentage and regions of each tumor were determined. Percentage of tumor cells staining was defined as complete absence of staining, $<50 \%$, or $>50 \%$ staining of the tumor composition. Regional staining patterns consisted of either a diffuse pan-tumoral pattern or a tumoral-stromal basilar interface staining pattern. Statistics were determined by the Student $t$ test with values of $<.05$ deemed significant. Variables statistically measured included mean immunohistochemical intensity scores for VCAM (CD-106), and ICAM (CD-54) among the various types of keratoacanthoma and squamous cell carcinoma.

\section{RESULTS}

The demographic features of the patients studied are shown in Table 1 . The average clinical follow-up was 2-6 years, ranging from 6 months to 4.2 years. Chart review failed to show any cases of metastasis or of death due to disseminated disease.

VCAM (CD-106) showed moderate diffuse cytoplasmic staining of the native dermal and subcutaneous fat vascular endothelium and dermal dendritic macrophages. Weak diffuse cytoplasmic staining of lymphocytes, eosinophils, and neutrophils was also observed. The epithelium of the adnexae and epidermis showed diffuse weak cytoplasmic staining.

ICAM (CD-54) showed moderately intense cytoplasmic membrane staining of the native endothelium, lymphocytes, and dermal dendritic cells. Other inflammatory cells including neutrophils and eosinophils showed weak membranous cytoplasmic staining. The adnexae and epidermis showed no staining.

The keratoacanthoma showed a variable pattern of VCAM (CD-106) and ICAM (CD-54) immunohistochemical intensity that was dependent upon the stage of tumor examined (Fig. 3). Both markers showed weak to absent staining in the early evolving and late resolving stages of the tumors. VCAM

TABLE 1. Demographic Features of Study

\begin{tabular}{|c|c|c|c|c|c|c|c|c|c|c|}
\hline \multirow{2}{*}{ Tumor } & \multicolumn{5}{|c|}{ Age } & \multicolumn{2}{|c|}{ Sex } & \multicolumn{3}{|c|}{ Location } \\
\hline & $<50$ & $51-60$ & $61-70$ & $71-80$ & $>80$ & $\mathrm{M}$ & $\mathrm{F}$ & Extremities & Trunk & Head and Neck \\
\hline Evolving KA & 0 & 3 & 2 & 3 & 2 & 8 & 2 & 6 & 3 & 1 \\
\hline Fully developed KA & 0 & 4 & 1 & 3 & 2 & 9 & 1 & 5 & 4 & 1 \\
\hline Resolving KA & 0 & 1 & 6 & 3 & 0 & 9 & 1 & 5 & 3 & 2 \\
\hline Well-differentiated SCC & 2 & 3 & 1 & 3 & 1 & 8 & 2 & 5 & 1 & 4 \\
\hline Poorly-differentiated SCC & 1 & 3 & 2 & 3 & 1 & 9 & 1 & 4 & 0 & 6 \\
\hline
\end{tabular}

TABLE 2. Staining Results

\begin{tabular}{|c|c|c|c|c|c|c|c|}
\hline \multirow{2}{*}{ Tumor } & \multicolumn{5}{|c|}{ Staining Intensity } & \multirow{2}{*}{ Percentage Cell Staining } & \multirow{2}{*}{ Pattern } \\
\hline & 0 & $1+$ & $2+$ & $3+$ & Average & & \\
\hline \multicolumn{8}{|l|}{ CD-54 results } \\
\hline Evolving KA & 8 & 2 & 0 & 0 & 0.2 & $<50 \%$ & Tumor-StromalInterface \\
\hline Fully developed KA & 0 & 5 & 5 & 0 & 1.5 & $<50 \%$ & Tumor-StromalInterface \\
\hline Resolving KA & 7 & 2 & 1 & 0 & 0.3 & $<50 \%$ & Tumor-StromalInterface \\
\hline Well-differentiated SCC & 0 & 1 & 8 & 1 & 2.0 & $<50 \%$ & Tumor-StromalInterface \\
\hline Poorly-differentiated SCC & 0 & 0 & 3 & 7 & 2.7 & $>50 \%$ & Diffuse \\
\hline \multicolumn{8}{|l|}{ CD-106 results } \\
\hline Evolving KA & 8 & 1 & 1 & 0 & 0.3 & $>50 \%$ & Diffuse \\
\hline Fully developed KA & 0 & 1 & 6 & 3 & 2.2 & $>50 \%$ & Diffuse \\
\hline Resolving KA & 8 & 2 & 0 & 0 & 0.2 & $>50 \%$ & Diffuse \\
\hline Well-differentiated SCC & 0 & 1 & 7 & 2 & 2.1 & $>50 \%$ & Diffuse \\
\hline Poorly-differentiated SCC & 0 & 0 & 7 & 3 & 2.9 & $>50 \%$ & Diffuse \\
\hline
\end{tabular}




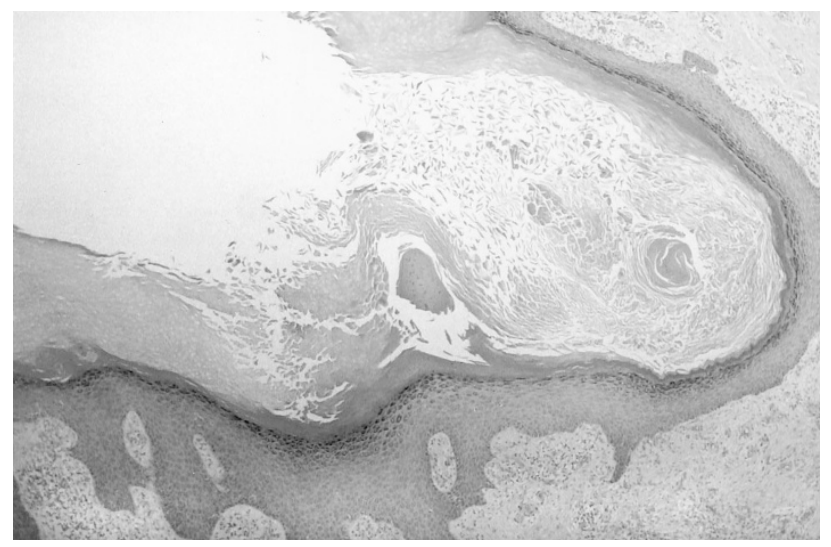

FIGURE 2. Resolving keratocanthoma with scalloped epithelial remnants punctuated by perforating strands of elastin fibers $(100 \times)$.

(CD-106) showed the greatest increase in intensity in the fully developed stage with an average intensity of $2.2 \pm 1.2$ for VCAM (CD-106) compared with $1.5 \pm 0.5$ for CD-54. There was a statistically significant difference in CD-106 and CD-54 expression in the fully developed keratoacanthoma compared to the evolving/resolved keratoacanthoma, $P=.01$ for CD-106 and $P=.03$ for CD-54. There was no difference in the level of intensity between marker expression (CD-106 and CD-54) in the fully developed keratoacanthoma $P=.67$. Greater than $50 \%$ of the lesional cells stained for CD-106 with less than $50 \%$ of the tumor cells stained for CD-54 (Table 2). The pattern of staining was diffusely cytoplasmic for VCAM (CD-106) and membranous for ICAM (CD-54). CD-106 showed pan-tumoral staining and CD-54 showed preferential staining of the stromaltumoral basilar epithelium (Fig. 4). The squamous cell carcinoma showed differential staining intensity depending upon the tumor grade. The well-differentiated squamous cell carcinoma showed an average intensity of $2.1 \pm 1.0$ and 2.0 \pm 1.0 for VCAM (CD-106) and ICAM (CD-54) respectively (Fig. 5). The intensity was increased at 2.9 \pm 0.7 and $2.7 \pm 0.7$ for VCAM (CD-106) and ICAM (CD-54) respectively among the poorly differenti-

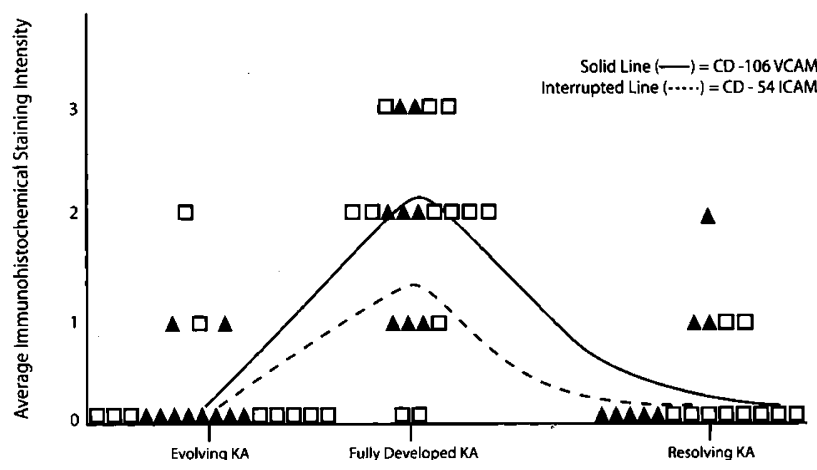

FIGURE 3. Keratocanthoma temporal expression pattern for CD-54 and $\mathrm{CD}-106$.

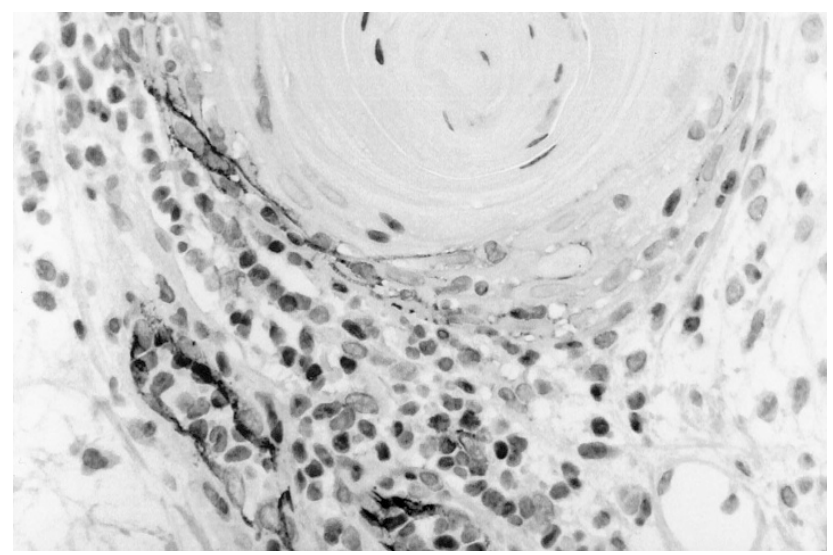

FIGURE 4. CD-54 immunostaining of well-developed keratocanthoma $(400 \times)$.

ated squamous cell carcinoma. Although there was statistically significant difference in the expression of CD-106 and CD-54 between the fully developed keratocanthoma and poorly differentiated squamous cell carcinoma, $P=.05 \mathrm{CD}-106, P=.02$ CD-54, there was no difference observed between the fully developed keratoacanthoma and welldifferentiated squamous cell carcinoma, $P=.81$ CD-106 and $P=.32$ CD-54. VCAM (CD-106) showed diffuse cytoplasmic staining compared with the membranous staining for ICAM (CD-54) in the squamous cell carcinoma. The patterns of staining showed greater than $50 \%$ of tumor cells stained throughout both carcinomas with CD-106 and the poorly differentiated squamous cell carcinoma with CD-54 (Table 2). CD-54 stained less than $50 \%$ of the tumor cells in well-differentiated squamous cell carcinoma. CD-106 showed pan-tumoral staining for both types of squamous cell carcinoma, with CD-54 showing a preferential tumor-stromal interface staining pattern in the well-differentiated squamous cell carcinoma and diffuse pan-tumoral staining in the poorly differentiated squamous cell carcinoma (Fig. 6).

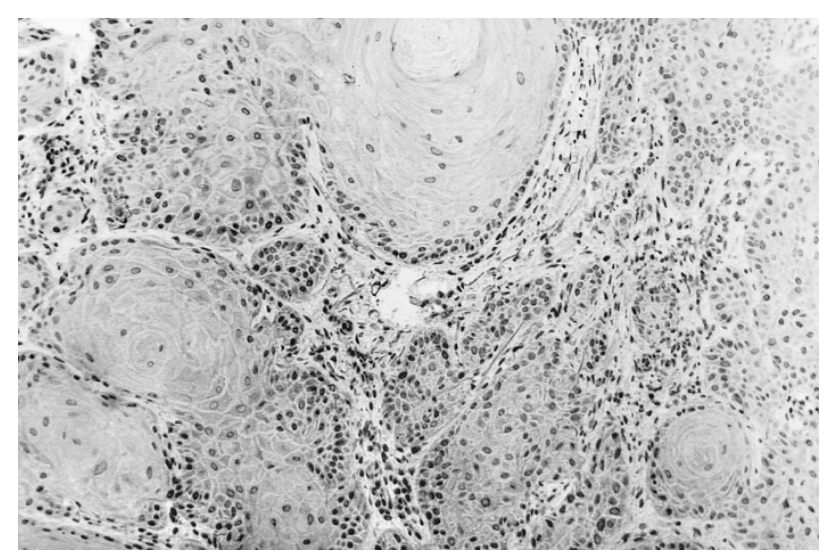

FIGURE 5. CD-106 immunostaining of well-differentiated squamous cell carcinoma $(400 \times)$. 


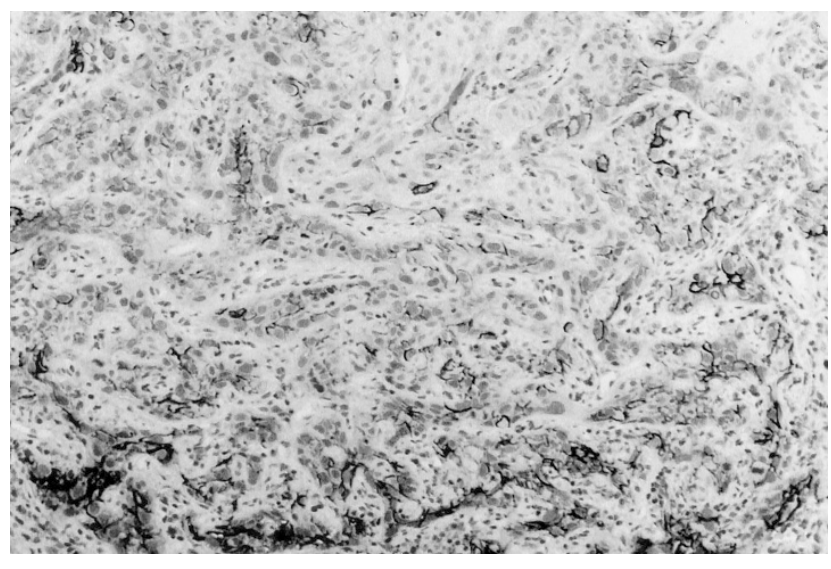

FIGURE 6. CD-54 immunostaining of poorly differentiated squamous cell carcinoma $(100 \times)$.

\section{DISCUSSION}

Keratoacanthoma are rapidly progressing distinct histological tumors which frequently regress within several months. Their histological marker is a keratin-filled central crater, surrounded by squamous epithelium (14). Cytologically they appear similar to squamous cell carcinoma (15). Although the biologic behavior of keratoacanthoma is usually characterized by rapidly growing neoplasia, with regression in several months, there are cases of metastases (3). This has led to the controversy over whether keratoacanthoma are a distinct entity or a variant of cutaneous squamous cell carcinoma (16, 17). In this study we have looked at two adhesion molecules in an attempt to explain the biologic behavior and pathogenesis of keratoacanthoma.

In order to understand the biologic behavior and pathogenesis of keratoacanthoma we examined both keratoacanthoma and cutaneous squamous cell carcinoma on their basis of immunohistochemical staining of two adhesion molecules. The majority of keratoacanthoma used in our study came from sun-exposed areas on elderly fair skinned individuals, which was consistent with the literature on clinical features. We excluded certain variants of the keratoacanthoma, including the giant keratoacanthoma, Ferguson-Smith, subungual and those associated with Muir Torre syndrome. Unlike previous studies, our study divided keratoacanthoma into the following groupings; evolving, fully developed and resolving. This allowed us to compare keratoacanthoma at different stages in their biological development with the well differentiated and poorly differentiated squamous cell carcinoma.

VCAM (CD-106) is a member of the immunoglobulin superfamily of adhesion molecules involved in cell-to-cell interaction. It is involved primarily in leukocyte and endothelial cell interactions and is expressed mainly on endothelial cells, regulating leukocyte migration across blood vessel walls (18).
VCAM (CD-106) is found especially at areas of inflammation and is inducible by a number of cytokines including IL- 1 and TNF- $\alpha$. ICAM (CD-54) is expressed on monocytes and endothelial cells in response to inflammatory cytokines, including TNF- $\alpha$. It is involved in the adhesion of neutrophils to the endothelium at the site of inflammation (12). In a recent study of keratoacanthoma and squamous cell carcinoma, TNF- $\alpha$ was shown to be equally expressed in both tumor types (19). This suggests that ICAM (CD-54) and VCAM (CD-106) would have equal expression in both tumor types. Our study has shown they do not. We show a seven fold increase in VCAM (CD-106) staining in fully developed keratoacanthoma over the evolving and involuting cases. This suggests a possible tumoral production of the adhesion molecules during this stage.

To date, VCAM (CD-106) has not been studied in keratoacanthoma. The study involving VCAM (CD-106) and cutaneous squamous cell carcinoma investigated its correlation with TNF- $\alpha$ in several epidermal tumors (20). In our study, we found VCAM (CD-106) to increase in conjunction with evolution of keratoacanthoma and decrease with involution. An increase was also seen in squamous cell carcinoma dedifferentiation. These results are commensurate with an increased inflammatory response seen in conjunction with cytokine elicited immune response in fully developed keratoacanthoma.

ICAM has been studied in cutaneous squamous cell carcinoma and keratoacanthoma and the results of our study were consistent with the previous study in that both tumors stain positive for the adhesion molecule (5). Our study differed in that we showed less percentage of tumor staining of the keratoacanthoma and a greater percentage of tumor staining of the squamous cell carcinoma than the previous study. This is interesting in that ICAM (CD54 ) is known to be up regulated in inflammatory dermatosis. It may be instructive that the strongest ICAM expression was found in fully developed lesions, at which point a brisk host response engenders the resolving phase of keratoacanthoma. As the inflammatory infiltrate subsides, there is a concomitant decrease in ICAM (CD-54) expression.

In conclusion, our results do not definitively answer the question as to the association between SCC and KA. However, temporal differences in VCAM (CD-106) and ICAM (CD-54) expression in keratoacanthoma may explain the observed differences in biologic behavior between these tumors. Further these results may offer some insights into the biologic course of keratoacanthoma. 


\section{REFERENCES}

1. Beham A, Regauer S, Soyer HP, Beham-Schmid C. Keratoacanthoma: a clinically distinct variant of well differentiated squamous cell carcinoma. Adv Anat Pathol 1998;5:269-80.

2. LeBoit PE. Is keratoacanthoma a variant of squamous cell carcinoma. New insights into an old controversy ... soon? Am J Dermatopathol 1995;4:319-20.

3. Hodak E, Jones RE, Ackerman AB. Solitary keratoacanthoma is a squamous-cell carcinoma: three examples with metastases. Am J Dermatopathol 1993;4:332-42.

4. LeBoit PE. Can we understand keratoacanthoma? Am J Dermatopathol 2002;2:166-8.

5. Patel A, Halliday GM, Cooke BE, Barnetson RS. Evidence that regression in keratoacanthoma is immunologically mediated: a comparison with squamous cell carcinoma. Br J Dermatol 1994;6:789-98.

6. Mukunyadzi P, Sanderson RD, Fan CY, Smoller BR. The level of syndecan-1 expression is a distinguishing feature in behavior between keratoacanthoma and invasive cutaneous squamous cell carcinoma. Mod Pathol 2002;1:45-9.

7. Murphy GF, Flynn TC, Rice RH, Pinkus GS. Involucrin expression in normal and neoplastic human skin: a marker for keratinocyte differentiation. J Invest Dermatol 1984;5:453-7.

8. Smoller BR, Kwan TH, Said JW, Banks-Schlegel S. Keratoacanthoma and squamous cell carcinoma of the skin: immunohistochemical localization of involucrin and keratin proteins. J Am Acad Dermatol 1986;2 Pt 1:226-34.

9. Van Der Laan N, de Leij LF, ten Duis HJ. Immunohistopathological appearance of three different types of injury in human skin. Inflamm Res 2001;7:350-6.

10. Haraldsen G, Kvale D, Lien B, Farstad IN, Brandtzaeg P. Cytokine-regulated expression of E-selectin, intercellular ad- hesion molecule-1 (ICAM-1), and vascular cell adhesion molecule-1 (VCAM-1) in human microvascular endothelial cells. J Immunol 1996;7:2558-65.

11. van de Stolpe A, van der Saag PT. Intercellular adhesion molecule-1. J Mol Med 1996;1:13-33.

12. Markovic S. Effects of cytokines on the expression of adhesion molecules on human umbilical vein endothelial cells (HUVECs). EJIFCC Vol. 13, no. 3. Available at: www.ifcc.org/ ejifcc/vol13no3/130301005.htm.

13. Bevilacqua MP. Endothelial-leukocyte adhesion molecules. Annu Rev Immunol 1993;11:767-804.

14. Kern WH, McCray MK. The histopathologic differentiation of keratoacanthoma and squamous cell carcinoma of the skin. J Cutan Pathol 1980;5:318-25.

15. Manstein CH, Frauenhoffer CJ, Besden JE. Keratoacanthoma: is it a real entity? Ann Plast Surg 1998;5:469-72.

16. Tran TA, Ross JS, Sheehan CE, Carlson JA. Comparison of oncostatin $\mathrm{M}$ expression in keratoacanthoma and squamous cell carcinoma. Mod Pathol 2000;4:427-32.

17. Tsuji T. Keratoacanthoma and squamous cell carcinoma: study of PCNA and Le(Y) expression. J Cutan Pathol 1997;7:409-15.

18. Bevilacqua MP, Nelson RM, Mannori G, Cecconi O. Endothelial-leukocyte adhesion molecules in human disease. Annu Rev Med 1994;45:361-78.

19. Lowes MA, Bishop GA, Cooke BE, Barnetson RS, Halliday GM. Keratoacanthomas have an immunosuppressive cytokine environment of increased IL-10 and decreased GM-CSF compared to squamous cell carcinomas. $\mathrm{Br} \mathrm{J}$ Cancer 1999;10:1501-5.

20. Viac J, Schmitt D, Claudy A. Expression of adhesion receptors in epidermal tumors: correlation with TNF alpha expressing cells. Anticancer Res 1995;2:551-5.

\section{Book Review}

\section{Carter D: Interpretation of Breast Biopsies, 4th Edition, 417 pp, Philadelphia, Lippincott, Wilkins \& Williams, 2002 (\$149.00).}

At 417 pages, the 4th edition of Darryl Carter's Interpretation of Breast Biopsies is more than double the size of the original edition, but the text is still handbook-sized and easy to digest. This edition also comes with a CD that contains more than 600 additional color pictures to supplement the textbook, which still has primarily black and white images.

The strength of this book over many of the other standard breast pathology books available today is that it incorporates the clinical correlation with pathologic interpretation. For example, there are chapters on risk factors in breast cancer (Chapter 2), biopsy and local treatment (Chapter 3 ), and prognosis and systemic therapy (Chapter 9). Even within the other chapters, there are nice discussions about clinically significant findings.

The CD is indeed helpful, in that lesions are illustrated more fully and in color. The illustrations on the CD are tied into the text by refer- ences to "efigures." There is also a "quiz" section, which presents unknowns and can be used for resident education or self-assessment. The pictures are somewhat variable in quality: some appear to have been scanned from old kodachromes, whereas others appear to be freshly taken, crisp images. The CD does not include text, except for legends. It appears somewhat flat, without much interactive capability. For example, pictures are presented at one size, without the ability to magnify areas that you wish to look at more closely. Nor can you call up the accompanying text to read more about the lesion or skip to other chapters that may discuss other aspects of a particular lesion. So for someone who has used e-texts, this certainly does not compare favorably.

\section{I-Tien Yeh \\ University of Texas Health Science Center at San Antonio \\ San Antonio, Texas}

